\title{
CONICAL QUANTUM BILLIARD REVISITED
}

\author{
BY \\ RICHARD L. LIBOFF \\ Schools of Electrical Engineering and Applied Physics and Center for Applied Math, \\ Cornell University, Ithaca, NY
}

\begin{abstract}
Eigenstates of a particle confined to a cone of finite length capped by a spherical surface element are derived. A countable infinite set of solutions is obtained corresponding to integer azimuthal and orbital quantum numbers $(m, l)$. These solutions apply to a discrete subset of the domain of half vertex angles, $0 \leq \theta_{0} \leq \pi / 2$. For arbitrary real orbital quantum numbers, $l \rightarrow \nu$, solutions are given in terms of the hypergeometric function, with $\nu=\nu\left(\theta_{0}\right)$, and are valid in the $\theta_{0}$ domain, $0 \leq \theta_{0}<\pi / 2$. Eigenstates are either nondegenerate or two-fold degenerate. Numerical examples of both classes of solutions are included. For the case $\mu=\cos \pi / 4$, the ground-state wavefunction and eigenenergy are

$$
\varphi_{G}=P_{\nu}(\mu) j_{\nu}\left(x_{\nu 1} r / a\right), \quad E_{G}=\hbar^{2}(6.4387)^{2} /\left(2 M a^{2}\right)
$$

where $\nu=2.54791, P_{\nu}(\mu)$ are Legendre functions, $x_{\nu 1}$ is the first finite zero of the spherical Bessel function $j_{\nu}(x), M$ is the mass of the confined particle and $a$ is the edgelength of the cone. Solutions constructed also represent the scalar $\hat{\mathbf{r}} \cdot \mathbf{E}$ electric field, where $\hat{\mathbf{r}}$ is the unit radius from the vertex of the cone. The first excited state of the conical quantum billiard has the nodal surface $\mu=1$ for all $0 \leq \mu_{0} \leq 1$.
\end{abstract}

1. Introduction. The quantum billiard problem refers to a configuration in which a particle is confined to a convex domain with perfectly reflecting walls [1]-[6]. This situation is described by the Helmholtz equation with Dirichlet boundary conditions. The present work stems from a previous analysis in which the circular sector quantum billiard was solved [2]. It was found that a critical vertex angle, $\theta_{\mathrm{c}}=0.354 \pi$, exists such that the nodal curve of the first excited state (equivalently, the second eigenstate of the Laplacian) is an arc for $\theta<\theta_{\mathrm{c}}$. For $\pi \geq \theta>\theta_{\mathrm{c}}$ the nodal is a line that bisects the vertex angle.

In the present study this configuration is generalized to the three-dimensional conical quantum billiard. This domain is a cone of finite length capped by a spherical surface element whose radius of curvature is the side-length, $a$, of the cone. The conical section has half vertex angle $\theta_{0}=\cos ^{-1} \mu_{0}$. In a previous study of this problem [7] solutions were

Received July 12, 1999.

2000 Mathematics Subject Classification. Primary 33C45, 35Q70, 35J25. 
obtained that satisfy the Helmholtz equation with Dirichlet boundary conditions. However, these solutions include associated Legendre functions of the second kind (of integer order) that are singular on the polar axis and therefore are not physically consistent. In the present work this configuration is revisited and physically and mathematically consistent eigenfunctions and eigenvalues are obtained, valid for conical sections with $0<\mu_{0} \leq 1$. A countable infinite subset of solutions is found corresponding to an integer orbital quantum number, $l$. For $l$ arbitrary and real $(l \rightarrow \nu)$, solutions are given in terms of hypergeometric functions and spherical Bessel functions. Elements of group theory [8] imply that eigenstates are either nondegenerate or two-fold degenerate. Numerical examples of both classes of solutions are given. A description of the hemisphere quantum billiard $\left(\mu_{0}=0\right)$ is included, and it is found that the first excited state of the conical quantum billiard has the nodal surface $\mu=1$ for all $0 \leq \mu_{0} \leq 1$. It is noted that this analysis applies as well to the scalar $\hat{\mathbf{r}} \cdot \mathbf{E}$ electric field, where $\hat{\mathbf{r}}$ is the unit radius from the vertex of the cone $[9]$.

\section{Analysis.}

2a. Nondegenerate integer $l$ solutions. Symmetries of the conical billiard are described by the $C_{\infty v}$ group, dimensions of whose irreducible representations indicate that eigenstates of the conical quantum billiard are one or two-fold degenerate. A subset of solutions for this problem may be obtained as follows. We work in spherical coordinates with the polar angle $\theta$ measured from the $z$-axis aligned with the axis of the cone, of a Cartesian $(x, y, z)$ frame. The azimuthal angle $\phi$ is measured from, say, the $x$-axis in the $(x, y)$-plane. Consider the solutions

$$
\begin{gathered}
\varphi_{n l}(r, \theta)=P_{l}\left(\mu, \mu_{0}\right) j_{l}\left(x_{l n} \frac{r}{a}\right), \\
\mu \equiv \cos \theta
\end{gathered}
$$

where $j_{l}(r)$ are spherical Bessel functions, [10], and

$$
j_{l}\left(x_{l n}\right)=0
$$

and $P_{l}(\mu)$ are Legendre polynomials. The function $P_{l}\left(\mu, \mu_{0}\right)$ has its first zero at $\mu=\mu_{0}$, so that $l=l\left(\mu_{0}\right)$ and $P_{l}\left(\mu_{0}, \mu_{0}\right)=0$.

The half vertex angle $\theta_{0}$ lies in the domain

$$
0 \leq \theta_{0} \leq \pi / 2 ; \quad 0 \leq \mu_{0} \leq 1
$$

Since the zeros of $P_{l}(\mu)$ functions $(l>1)$ comprise a subset of algebraic numbers in $(0,1)$, the solutions (1a) are valid for a subset of the conical $\mu_{0}$ values (1d). Eigenenergies corresponding to (1a) are given by

$$
E_{n l}=\frac{\hbar^{2} x_{l n}^{2}}{2 M a^{2}} \propto\langle\ln |\Delta| \ln \rangle,
$$

where $M$ is the mass of the confined particle and $\Delta$ is the Laplacian and $\langle\mid\rangle$ represents an integral inner product over the conical domain [10]. Since the conical surface of the given configuration is a nodal surface of an eigenstate of the spherical quantum billiard, the eigenenergies (2) are likewise eigenenergies of the spherical quantum billiard. In the 
limit, $\mu_{0} \rightarrow 1\left(\theta_{0} \rightarrow 0\right)$, higher $l$ values are required to satisfy $P_{l}\left(\mu, \mu_{0}\right)=0$. This implies that $x_{l n}$ grows as $\mu_{0} \rightarrow 1$ and, consistently with (2), energy grows larger in this limit.

At a given value of $l=l\left(\mu_{0}\right)$, with Courant's nodal partitioning theorem, [11], the ground state is the non-nodal state, given by

$$
\varphi_{1 l}(r, \theta)=P_{l}\left(\mu, \mu_{0}\right) j_{l}\left(x_{l 1} \frac{r}{a}\right) \text {. }
$$

Higher excited states are given by (1a) and have spherical nodals at $r<a$, corresponding to higher zeros of $j_{l}(x)$. First we consider eigenstates corresponding to the values $l>1$. Here is a list of the first few $P_{l}(\mu)$ functions:

$$
\begin{gathered}
P_{0}(\mu)=1, \quad P_{1}(\mu)=\mu, \quad P_{2}(\mu)=\frac{1}{2}\left(3 \mu^{2}-1\right), \\
P_{3}(\mu)=\frac{1}{2}\left(5 \mu^{3}-3 \mu\right), \quad P_{4}(\mu)=\frac{1}{8}\left(35 \mu^{4}-30 \mu^{2}+3\right) .
\end{gathered}
$$

Thus, for example, the finite zero of $P_{3}(\mu)$ describes a cone with half angle

$$
\theta_{0}=\cos ^{-1} \sqrt{3 / 5} \cong \frac{\pi}{4.56}
$$

corresponding to the solution

$$
\varphi_{n 3}=P_{3}\left(\mu, \sqrt{\frac{3}{5}}\right) j_{3}\left(x_{3 n} \frac{r}{a}\right) .
$$

The eigenstates (1a) comprise a countable infinite series of solutions corresponding to a given $l$ value and an infinite number of $x_{l n}$ values. This procedure may be extended. Thus, a second countable infinite set of solutions is given by $P_{l, 2}\left(\mu, \mu_{0}\right), l>2$. These functions have their first zero in the domain $0<\mu<1$ and their second zero at $\mu=\mu_{0}$. The functions $P_{l, n}\left(\mu, \mu_{0}\right), l>n$ have their $n$th zero at $\mu=\mu_{0}$. Again with reference to Courant's nodal partitioning theorem, the eigenenergy corresponding to $P_{l,(n+j)}\left(\mu, \mu_{0}\right)$ is larger than that associated with $P_{l, n}\left(\mu, \mu_{0}\right)(l>n, j>0$ and integer). The solutions $P_{l}(\mu, 0)$, (for odd $l$ ) correspond to the hemisphere quantum billiard. Specifically, $P_{1}(\mu, 0)=P_{1}(\mu)=\mu$.

2b. Degenerate integer $l$ solutions. The preceding technique may be extended to associate Legendre functions $P_{l}^{m}(\mu)$. We recall the series representation of these functions [12]:

$$
\begin{aligned}
P_{l}^{m}(\mu)=A_{l m}\left(1-\mu^{2}\right)^{m / 2}\left\{\mu^{l-m}-\right. & \frac{(l-m)(l-m-1)}{2(2 l-1)}\left[\mu^{l-m-2}\right. \\
& \left.\left.-\frac{(l-m-2)(l-m-3)}{4(2 l-3)} \mu^{l-m-4}+\cdots\right]\right\},
\end{aligned}
$$

where $A_{l_{m}}$ is a constant coefficient. It follows that $P_{l}^{m}(\mu)$ has zeros in the open $\mu$ interval, $(0,1)$, provided that $l-m \geq 2$. For such cases, following the procedure above, solutions to the conical quantum billiard, corresponding to the cone angle, $\mu_{0}$, are written $P_{l}^{m}\left(\mu, \mu_{0}\right)$. Since $m>0$ these eigenfunctions are two-fold degenerate, corresponding to the two values, $\pm m$. We recall that $P_{l}^{ \pm m}(\mu)$ have the same zeros [see (5b) below].

An example of this formalism is given by the associated Legendre function

$$
P_{3}^{1}(\mu)=\frac{3}{2}\left(1-\mu^{2}\right)^{1 / 2}\left(5 \mu^{2}-1\right),
$$


which has a zero at $\mu=\sqrt{1 / 5}$ corresponding to the cone angle

$$
\theta_{0}=\cos ^{-1} \sqrt{1 / 5} \approx \frac{\pi}{2.84}
$$

These values give the two-fold degenerate eigenfunctions

$$
\varphi_{3, n, \pm 1}(r, \mu, \phi)=P_{3}^{1}(\mu, \sqrt{1 / 5}) \exp ( \pm i \phi) j_{3}\left(x_{3 n} r / a\right) .
$$

The related eigenenergy is given by (2). Generalizations of this formalism follow that given above for nondegenerate eigenstates. Due to lack of spherical symmetry of the conical quantum billiard, the value $P_{l}^{m}\left(\mu_{0}, \mu_{0}\right)=0$ does not imply, for example, that $P_{l}^{m^{\prime}}\left(\mu_{0}, \mu_{0}\right)=0$, where $\left|m^{\prime}\right| \neq|m|$. Thus, eigenstates are two-fold degenerate, in accord with group-theoretical stipulations. Similarly, nondegenerate eigenstates $P_{l}\left(\mu, \mu_{0}\right)$ found above do not generalize to associated Legendre function solutions for the same reason.

3. Arbitrary $l$ solutions. We seek solutions of the conical quantum billiard that are regular for all cone angles $0<\mu_{0} \leq 1,[12]-[15]$. As rotational symmetry is maintained about the $z$-axis, $m$-values remain integers. However, due to the break in $\theta$ symmetry, $\nu$-values are arbitrary and real, where we have set $l \rightarrow \nu$. We recall that the associated Legendre (second-order, ordinary) differential equation has three regular singularities, $(\mu \pm 1), \infty$, with related exponents, $(m / 2,-m / 2)^{2},(\nu+1,-\nu)$. At given values of $(\nu, m)$, Legendre's equation has two solutions. For $m>0$ and integer, the solution regular in the domain $0<\mu \leq 1$ is given by, [13],

$$
P_{\nu}^{m}(\mu)=\frac{1}{m !}\left(\frac{\mu-1}{\mu+1}\right)^{m / 2} \frac{\Gamma(\nu+m+1)}{\Gamma(\nu-m+1)} F\left(-\nu, \nu+1 ; 1+m ; \frac{1-\mu}{2}\right) \text {. }
$$

At $\mu=1, F=1$ and $P_{\nu}^{m}(1)=0$. When $\nu$ is an integer, $F$ becomes a polynomial and the latter equality maintains. Note also that $|\Gamma(z)|>0$ for all $z$. For integer $m<0$,

$$
P_{\nu}^{-m}(\mu)=(-1)^{m} \frac{\Gamma(\nu-m+1)}{\Gamma(\nu+m+1)} P_{\nu}^{m}(\mu)
$$

The hypergeometric function, $F$, is given by, [11]-[15],

$$
F(a, b ; c ; z)=\sum_{n=0}^{\infty} \frac{(a)_{n}(b)_{n}}{(c)_{n}} \frac{z^{n}}{n !}=1+\sum_{n=1}^{\infty} \frac{(a)_{n}(b)_{n}}{(c)_{n}} \frac{z^{n}}{n !}
$$

where the Pochhammer symbol

$$
(a)_{n} \equiv \frac{\Gamma(a+n)}{\Gamma(a)}=(a+n-1) \cdots(a)
$$

It follows that $F(a, b ; c ; 0)=1$ and that $F(a, b ; c ; z)=F(b, a ; c ; z)$. The solution (5a) is regular in the domain $0<\mu \leq 1$ or, equivalently, $0 \leq \theta<\pi / 2$. 
3a. The hemisphere quantum billiard $\left(\mu_{0}=0\right)$. Solutions for the hemisphere quantum billiard are given in terms of associated Legendre functions and spherical Bessel functions of integer order with $0 \leq|m| \leq l$. With the definition of $P_{l}^{m}(\mu)$ one finds the two solutions

$$
\begin{aligned}
& P_{l}^{m}(\mu)=\mu\left(1-\mu^{2}\right)^{m / 2} f\left(\mu^{2}\right), \quad(l-m) \text { odd, } \\
& P_{l}^{m}(\mu)=\left(1-\mu^{2}\right)^{m / 2} g\left(\mu^{2}\right), \quad(l-m) \text { even, }
\end{aligned}
$$

where $f$ and $g$ are polynomials and $g(0) \neq 0$. It follows that the boundary condition $\varphi_{n l}(\mu=0)=0$, relevant to the hemisphere quantum billiard, is satisfied by (5e). For this case, the first excited state has the quantum numbers $(l=1, m=0)$. Both equalities (5e, f) again return the property, $P_{l}^{m}(1)=0$. One may conclude that for all $\nu$ and related integer $m$, solutions of the conical quantum billiard exist for which $\mu=1$ is a nodal surface for $0 \leq \mu_{0} \leq 1$.

3b. Boundary conditions. As noted above, group properties of the conical quantum billiard imply that eigenstates are either nondegenerate or two-fold degenerate. Combining this property with symmetries of the cone imply that in any state of finite $\nu$, angular momentum of the state has two projections, $m= \pm m_{\nu}^{(n)}$, where $m_{\nu}^{(n)}$ is the largest integer less than or equal to $(\nu-n)$, where $n<\nu$ is an integer. This property is in accord with the case for integer $\nu=l$, for which $m$ decreases in integer steps from $l$ to $-l$.

With reference to $(5 \mathrm{a})$, boundary conditions correspond to the relation

$$
F\left(-\nu, \nu+1 ; 1+m_{\nu}^{(n)} ; \frac{1-\mu_{0}}{2}\right) \equiv F\left(\nu, n, \mu_{0}\right)=0 .
$$

In this technique of solution one looks for zeros of $(6 \mathrm{a})$ at $n=0,1$, etc. until a zero appears. This fixes $\nu$ and $m_{\nu}^{(n)}$ which, when substituted into (5a), give the $\mu$-component eigenfunction. In the event of degeneracy, the two solutions correspond to the values $\pm m_{\nu}^{(n)}$. Note that with (5b), $P_{\nu}^{ \pm m_{\nu}^{(n)}}(\mu)$ have the same zeros. The relation (6a) implies that the Legendre-function index, $\nu$, is an implicit function of $\mu_{0}$.

Over the domain of application, $1-\mu_{0} \geq 0$. In addition, $\Gamma(z)<0$ in intervals

$$
-(2 s+1)<z<-2 s,
$$

where $s \geq 0$ and is an integer. It follows that a solution to (6a) exists for selective $n$ values. Thus, the general solution to the conical quantum billiard is given by the two-fold degenerate functions

$$
\varphi_{n^{\prime} \nu m_{\nu}^{(n)}}(r, \theta, \phi)=P_{\nu}^{m_{\nu}^{(n)}}(\mu) \exp i\left( \pm m_{\nu}^{(n)} \phi\right) j_{\nu}\left(x_{\nu n^{\prime}} \frac{r}{a}\right)
$$

where $n^{\prime}$ is an integer. The corresponding eigenenergy is given by

$$
E_{n^{\prime} \nu}=\frac{\hbar^{2} x_{\nu n^{\prime}}^{2}}{2 M a^{2}}
$$

which, as in the integer $\nu$ case, is independent of the azimuthal $m_{\nu}$ quantum number.

3c. Spherical Bessel functions. We consider next, spherical Bessel functions of real order, and $2 \nu \neq$ integer, defined by

$$
j_{\nu}(z)=\sqrt{\frac{\pi}{2 z}} J_{\nu+1 / 2}(z)
$$




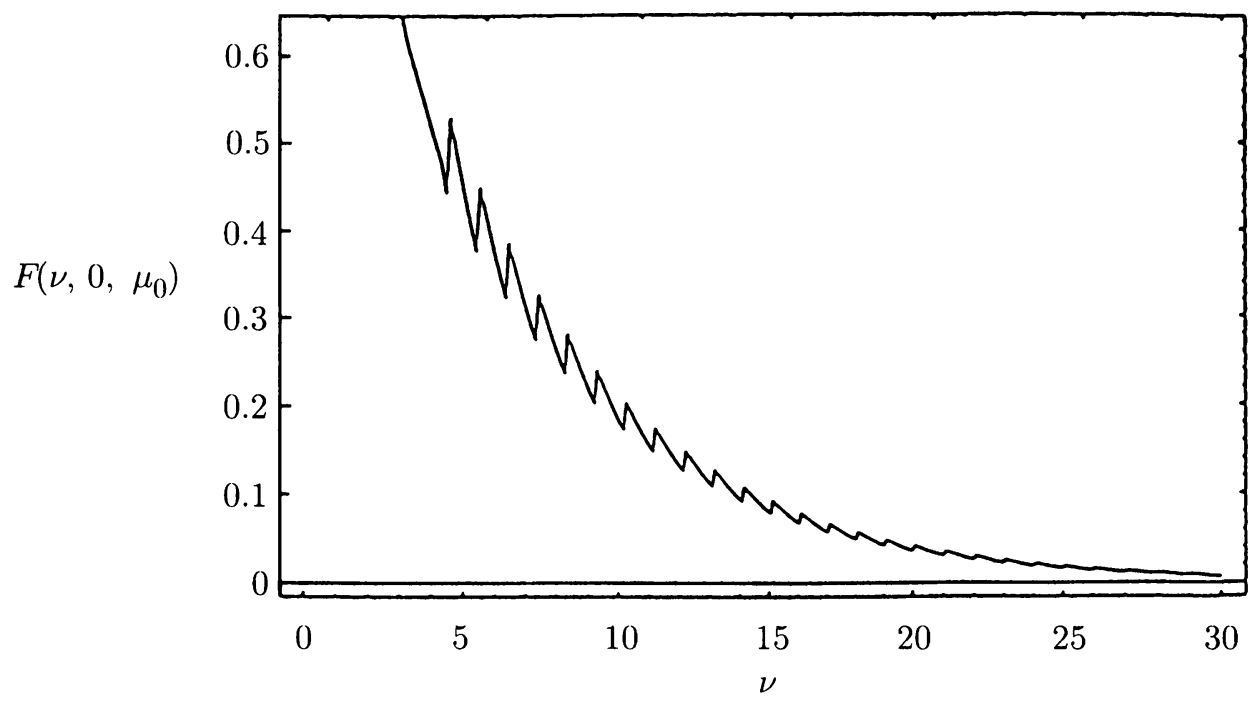

FIG. 1(a). At given $\mu_{0}$, roots of $F\left(\nu, n, \mu_{0}\right)$ determine $\nu$ and $m_{\nu}^{(n)}$. At $\mu_{0}=0.14565, F\left(\nu, 0, \mu_{0}\right)$ shows no zeros.

where $J_{\nu}(z)$ are Bessel functions of the first kind,

$$
J_{\nu}(z)=\sum_{q=1}^{\infty} \frac{(-1)^{q}}{q ! \Gamma(\nu+q+1)}\left(\frac{z}{2}\right)^{2 q+\nu} .
$$

3d. Examples. We choose

$$
\mu_{0}=1 / \sqrt{2} \quad\left(\theta_{0}=\pi / 4\right) ; \quad\left(1-\mu_{0}\right) / 2=0.14565
$$

and plot $F\left(\nu, n, \mu_{0}\right)$ against $\nu$ at given $n$ starting with $n=0$. For the case in point, $n=0,1$ give no zeros (Figs. 1a, b). At $n=2$, the zero $\nu=2.5479$ is obtained (Fig. 1c). (The second zero corresponds to higher energy.) It follows that $\nu-n=\nu-2=0.5479$ and $m_{\nu}^{(2)}=0$. The related wavefunction is nondegenerate. The first finite zero of the spherical Bessel function ( $7 \mathrm{~b})$ is $x_{\nu 1}=6.4387$ corresponding to the eigenenergy

$$
E_{0}=\frac{\hbar^{2}(6.4387)^{2}}{2 M a^{2}}
$$

This eigenenergy corresponds to the eigenfunction

$$
\varphi_{1 \nu}(r, \theta)=P_{\nu}(\mu) j_{\nu}\left(x_{\nu 1} \frac{r}{a}\right)
$$

where $\nu=2.54791$. Since there are no $m=0$, integer- $\nu$ solutions corresponding to $\mu_{0}=1 / \sqrt{2}$, (8c) represents the ground state of this quantum billiard. Each root of $F\left(\nu, n, \mu_{0}\right)$ represents a distinct eigenfunction and eigenenergy with corresponding $m_{\nu}^{(n)}$ numbers in (6c). For $m_{\nu}^{(n)}>0$, states are two-fold degenerate.

Our second example is the case $n=3$ corresponding to which there are three solutions (Fig. 1d). The second zero of $F\left(\nu, 3, \mu_{0}\right)$ is $\nu=4.40533$, for which $\nu-n=1.40533$, which gives $m_{\nu}^{(3)}=1$; so this eigenstate is doubly degenerate. The first finite zero of $j_{\nu}(x)$ for 


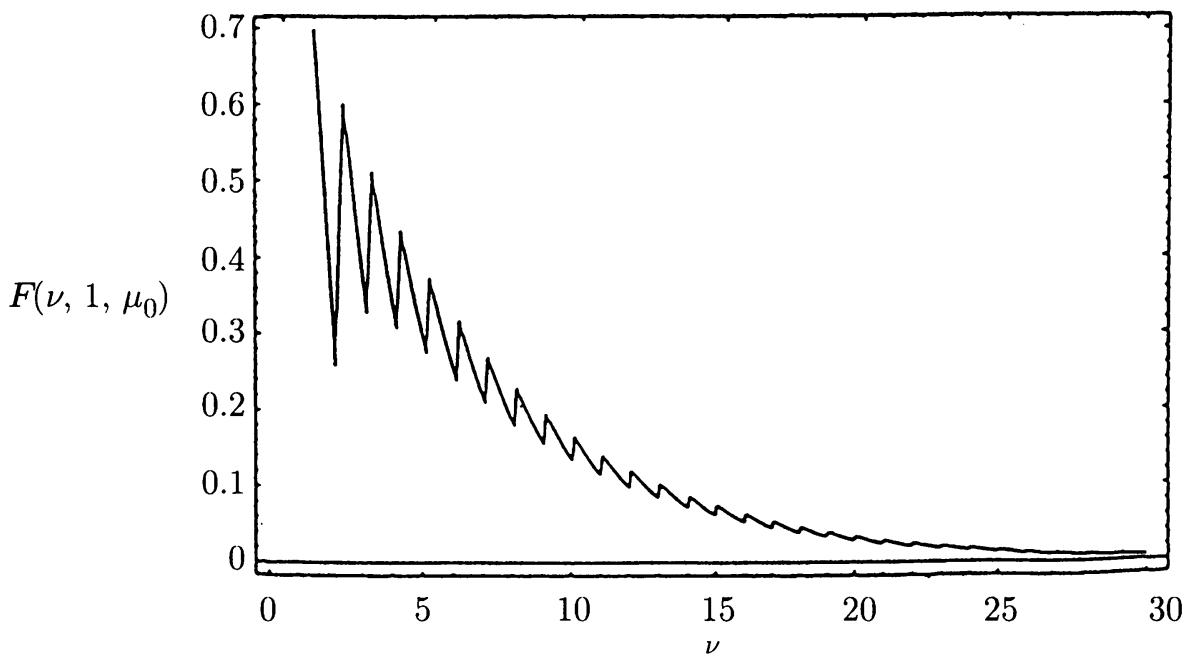

FIG. 1(b). At given $\mu_{0}$, roots of $F\left(\nu, n, \mu_{0}\right)$ determine $\nu$ and $m_{\nu}^{(n)}$. At $\mu_{0}=0.14565, F\left(\nu, 1, \mu_{0}\right)$ shows no zeros.

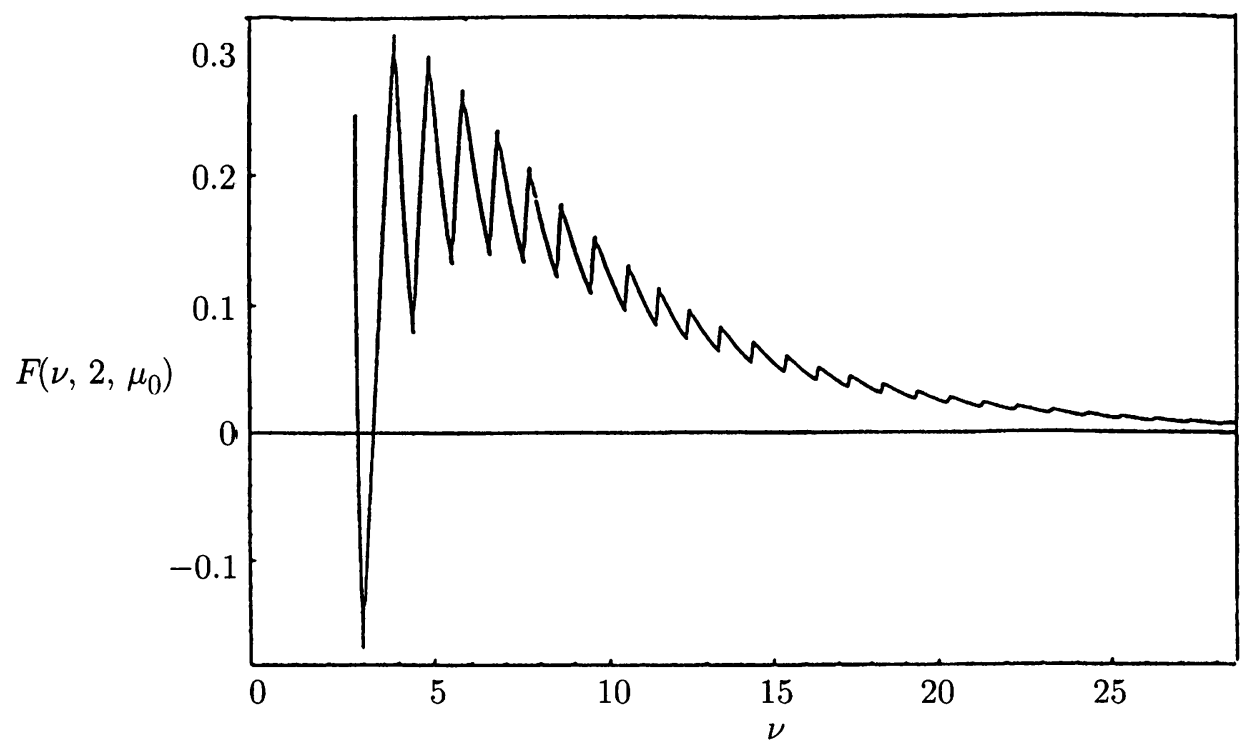

FIG. 1(c). At given $\mu_{0}$, roots of $F\left(\nu, n, \mu_{0}\right)$ determine $\nu$ and $m_{\nu}^{(n)}$. At $\mu_{0}=0.14565, F\left(\nu, 2, \mu_{0}\right)$ shows two zeros.

this example is $x_{\nu 1}=8.66035$ corresponding to the eigenenergy

$$
E_{3,1}=\frac{\hbar^{2}(8.66035)^{2}}{2 M a^{2}}
$$

and wavefunction

$$
\varphi_{3,1}(r, \mu, \phi)=P_{\nu}^{1}(\mu) \exp i\left(m_{\nu} \phi\right) j_{\nu}\left(x_{\nu 1} \frac{r}{a}\right)
$$




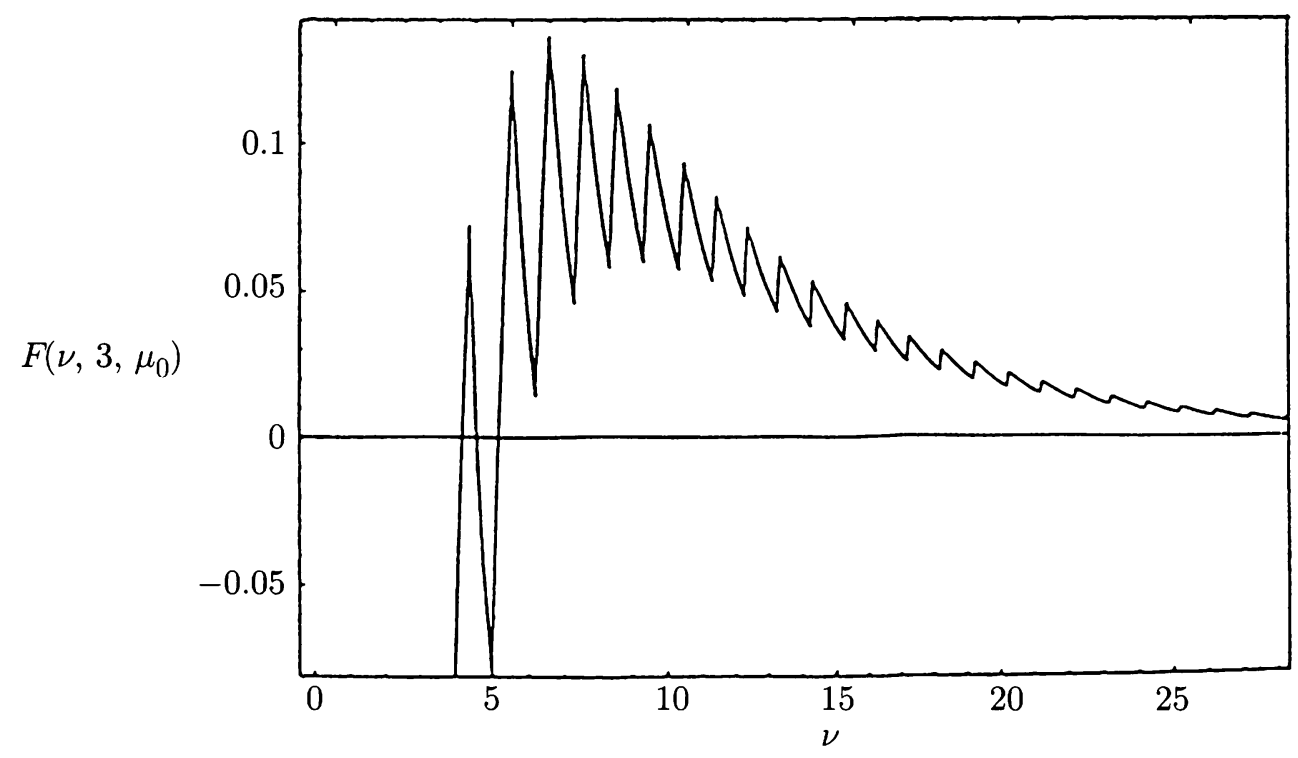

FIG. 1(d). At given $\mu_{0}$, roots of $F\left(\nu, n, \mu_{0}\right)$ determine $\nu$ and $m_{\nu}^{(n)}$. At $\mu_{0}=0.14565, F\left(\nu, 3, \mu_{0}\right)$ shows three zeros.

where $m_{\nu}= \pm 1$.

Conclusions. Eigenfunctions and eigenvalues of a particle confined to the interior of a cone capped by a spherical surface element are derived. A countable infinite subset of these solutions is composed of associate Legendre functions of integer azimuthal $\mathrm{m}$ numbers and integer orbital quantum $l$-numbers. For arbitrary real orbital quantum numbers, a continuum of solutions is derived, given in terms of the hypergeometric function, valid in the domain $0 \leq \theta_{0}<\pi / 2$, where $\theta_{0}$ is the half angle of the cone. In this case, the index $\nu$ of the Legendre function depends on $\theta_{0}$. Elements of group theory come into play in construction of these solutions.

Numerical examples are given for both classes of solutions. Solutions constructed also represent the scalar $\hat{\mathbf{r}} \cdot \mathbf{E}$ electric field, where $\hat{\mathbf{r}}$ is the unit radius from the vertex of the cone. A description of the hemisphere quantum billiard is included, from which it was concluded that $\mu=1$ is the nodal surface of the first excited state for all $0 \leq \mu_{0} \leq 1$.

Acknowledgments. Fruitful discussions of these topics with my colleagues Kurt Gottfried, John Hubbard, Larry Edwards, Gregory Ezra, Hercules Nevis, Michael Booth, Louis Hand, Clifford Pollack, Richard Friedberg and Paul McIsaac are gratefully acknowledged. I am especially grateful to Nils Weimann for numerical assistance in this problem and to Mason Porter for his careful reading of this manuscript. 


\section{REFERENCES}

[1] R. L. Liboff, The polygon quantum billiard problem, J. Math. Phys. 35, 596-607 (1994)

[2] R. L. Liboff, Circular-sector quantum billiard and allied configurations, J. Math. Phys. 35, 22182228 (1994)

[3] G. Alessandrini, Nodal lines of the fixed membrane problem in general convex domains, Comm. Math. Helv. 69, 142-154 (1994)

[4] M. A. Pinsky, The eigenvalues of an equilateral triangle, Siam J. Math. Anal. 11, 819-849 (1980)

[5] A. Melas, On the nodal line of the second eigenfunction of the Laplacian in $\mathbb{R}^{2}$, J. Differential Geom. 35, 255-263 (1992)

[6] J. B. Keller and S. I. Rubinow, Asymptotic solution of eigenvalue problems, Ann. Phys. 9, 24-75 (1960)

[7] R. L. Liboff, Conical quantum billiard, Letts. Math. Phys. 42, 389-391 (1997)

[8] F. A. Cotton, Chemical Application of Group Theory, Wiley, 3rd ed., New York, 1990

[9] J. D. Jackson, Classical Electrodynamics, 2nd ed., Sec. 16.35, Wiley, New York, 1975

[10] R. L. Liboff, Introductory Quantum Mechanics, 3rd ed., Section 10.3, Addison Wesley, Menlo Park, CA, 1998

[11] R. Courant and D. Hilbert, Methods of Mathematical Physics, Vol. 1, Wiley Interscience, New York, 1966

[12] E. Jahnke and F. Emde, Tables of Functions, 4th ed., Chap. VII, Dover, New York, 1945

[13] Z. X. Wang and D. R. Guo, Special Functions, World Scientific, Teaneck, NJ, 1984

[14] W. Magnus and F. Oberhettinger, Formulas and Theorems for the Special Functions of Mathematical Physics, Chelsea, New York, 1949

[15] M. Abramowitz and I. Stegun, Higher Transcendental Functions, Dover, New York, 1964 